\title{
El sitio arqueológico de Lagartero, Chiapas
}

\author{
Sonia Rivero Torres
}

Resumen: En este artículo se presenta la descripción del sitio de Lagartero, Chiapas, incluidas las diferentes islas que lo forman. Se continúa con los trabajos arqueológicos realizados desde los comienzos del proyecto de investigación en la isla Limonar, el cual tuvo lugar desde 1990, en el que se realizó la primera temporada de campo, y asi sucesivamente explicando los principales aspectos y hallargos arqueológicos en cada temporada (1993, 94, 95, 96, 97, 99, 2000, 2001) hasta el 2003, en el que se realizó la última fase de la octava temporada de campo. Con base en los materiales arqueológicos obtenidos se da una cronología relativa del sitio sustentada tanto en la tipología cerámica como por el C14. También conocemos la lengua que se habló en el sitio gracias a las investigaciones a partir de la Lingüistica Histórica. La función particular del sitio se debió a su localización privilegiada donde se pudo explotar tanto el medio ambiente terrestre como el lacustre, lo cual quedó plasmado tanto en su producción alfarera, como en el intercambio de la lítica y en un tipo particular de arquitectura.

Palabras clave: sitio arqueológico de Lagartero, Chiapas, trabajo arqueológico.

Enviado a dictamen: 27 de marzo de 2006.

Aprobación: 13 de febrero de 2007.

Sonia Evangelina Rivero Torres, doctora por la Universidad de Cambridge, Inglaterra, Dirección de Estudios Arqueológicos del Instituto Nacional de Antropología e Historia; Temas de especialización: análisis del patrón de asentamiento prehispánico en la cuenca superior del río Grijalva y la investigación, excavación y consolidación del sitio arqueológico de Lagartero, municipio de La Trinitaria, Chiapas, correo electrónico: sonia_rivero_torres@hotmail.com.
Abstract: This article talks about the archaeological site of Lagartero, Chiapas. That means it includes a description of all the various islands that form it. These islands have different sizes, but the biggest one is the Limonar island, where I have been working. You can find here the ceremonial area of the site. Later I described the archaeological works made since 1990, where I did the first field work on the L imonar island and then periodically continued excavating. L ater on I explained the relevant architectural aspects and archaeological founds in each field season (1993, 94, 95, 97, 99 and 2002) until to 2003, where I did the last part of the eighth field work. Supported by the archaeological data obtained in these field seasons we have a relative chronology of the site based on the ceramic typology and C14 analysis. We know about the language that was spoken in the area thanks to the research of the Historical Linguistic. The particular function of the site in its mayor apogee was due to its privileged location in the Lagartero marsh that means a lot of water and fertile soils. The people who inhabited Lagartero exploited both sources and caused a prosperity reflected in the material production, lithic exchange and a particular architectural style.

Key words: archaeological site of Lagartero, Chiapas, the archaeological work.

\section{Localización}

7 ste sitio se encuentra en el municipio de 1 La Trinitaria, entre 600 y $650 \mathrm{msnm}$, en Una planicie que se extiende hasta los Cuchumatanes guatemaltecos (figura 1). Para llegar se debe ir por la carretera Panamericana 
de Comitán a Ciudad Cuauhtémoc, y en el kilómetro 59, en Chamic, está la desviación rumbo a los Lagos de Colón; desde la que deben recorrerse otros 11.4 kilómetros hasta el ejido donde su ubican estos lagos. Hasta aquí la carretera está completamente pavimentada. Después se sigue un camino de tierra entre la milpa y la selva caducifolia. Después de pasar por varios arroyos bajos, se llega a la zona conocida localmente como Lagartero, la cual se halla esparcida dentro de este tipo de vegetación a más o menos tres kilómetros de distancia del ejido Cristóbal Colón (foto 1).

El sitio recibió este nombre por la ciénega de Lagartero, que tiene un área aproximada de 8.5 kilómetros cuadrados, alimentada por manantiales locales y las aguas de los ríos Lagartero y San Lucas, afluentes superiores del río Grijalva, por lo que no son aguas estancadas sino que corren de Este a Oeste (foto 2).

La ciénega de Lagartero está situada sobre estratos marinos del Mesozoico Superior y el Terciario Inferior Medio. El subsuelo está formado por rocas calizas que propician terrenos cársticos de relieve variado con agujeros, dolinas y hendiduras en forma de cráteres (Müllerried 1957: 130-131), lo que explica la formación de los Lagos de Colón como cenotes a diferentes niveles de tamaños no constantes por el desprendimiento continuo de cal, que se deposita en el fondo.

\section{Antecedentes arqueológicos}

El primer reconocimiento fue hecho por Thomas Lee (1974) durante su recorrido por la cuenca superior del río Grijalva, y lo identificó como un sitio de tipo ceremonial con el número Tr. 99. Posteriormente, la arqueóloga
Susan Ekholm hizo un recorrido de superficie en el sitio y excavó dos pozos estratigráficos en el montículo más grande, dentro de la plaza principal, en el área ceremonial, y encontró varios tipos de entierros. Además, hizo una trinchera al sur del montículo, que todavía tiene una gran ceiba, de donde obtuvo fragmentos de cerámica policroma en buen estado y figurillas elaboradas en barro con molde, como también gran cantidad de tepalcates monocromos. En 1976 regresó al sitio y excavó pozos de prueba en diferentes partes de la isla principal (El Limonar); halló material cerámico con una periodicidad del Clásico Medio (600-700 d. C.) al Posclásico (900-1521 d. C.) y en uno de los pozos dio con gran cantidad de tepalcates, por lo que ella (1979b:149) cree que esta zona fue utilizada como basurero. Además de los tepalcates, Ekholm encontró quinientos fragmentos de figurillas de arcilla hechas con molde, ornamentos de barro, instrumentos musicales, incensarios, huesos de animal, algunos huesos humanos, instrumentos en hueso, ornamentos de concha, gran cantidad de moluscos comestibles, navajas de obsidiana y gran variedad de artefactos de piedra, cuyo registro, por desgracia, todavía no ha sido publicado, con excepción de las figurillas y la descripción de la vasijas más importantes (Ekholm, 1979 y 1985). Entre los tepalcates policromos se pudo identificar platos pertenecientes a los horizontes Tepeu 1 y 2 (Willey, Culbert y Adams, 1967:289-305) y vasos cilíndricos con decoración de glifos - también pertenecientes a este período-, grandes ollas policromas, ollas miniatura y tambores. Esta cerámica presenta relación con o influencia de las tierras altas de Guatemala, en especial con las regiones Quiché y Alta Verapaz. 
La mayoría de la cerámica encontrada es monocroma, entre la que destacan ollas de cuellos altos con asas, ollas con cuellos efigie que representan animales, vasijas con bordes hacia dentro, platos y cajetes. A veinte metros al este de este basurero se localizaron cincuenta entierros (en la plaza noroeste) con ofrendas de cerámica y ninguna figurilla (Gurr, 1987).

Eduardo Martínez (1983) elaboró parte del mapa que Deann Gurr trazó en su segunda temporada de campo, así, completó la parte ceremonial (dos terceras partes del sitio), y sólo faltó por hacer la topografía de las partes norte y oeste (zona habitacional) y otra que antiguamente estuvo debajo del lago, con casas alrededor y que actualmente es milpa, como la probable entrada al sitio. Él terminó de hacer el levantamiento topográfico total del sitio (figura 2), que se tomó como base para trazar la actual poligonal de la zona arqueológica.

\section{Descripción del sitio Lagartero}

La mayor parte del sitio arqueológico está localizada en pequeñas islas y penínsulas que se proyectan dentro de los lagos. Lagartero fue un gran centro cívico-religioso, cuyo núcleo ceremonial se encuentra en una de las islas más grandes conocida localmente como El Limonal (Limonar), en la parte sur de la ciénega (figura 3), y que posiblemente fue un centro rector de la región durante el Clásico Tardío (700-900 d. C.). Está rodeado de agua, con unos lados más profundos que otros, y eso depende de si es temporada de lluvias o de secas. En esta isla hay 186 montículos de diferentes tamaños; son más o menos quince las construcciones principales; algunos no llegan a los treinta centímetros de altura y el más alto mide dieciséis metros. Hay un juego de pelota ya excavado, cuatro pirámides - dos ya exploradas que limitan la plaza principal-, plataformas de diferentes tamaños conectadas y aisladas, restos de estructuras sobre plataformas y basamentos de casas habitación, algunos ya liberados.

Las estructuras cívico-religiosas se encuentran alrededor de las plazas y los basamentos de casas forman patios, los cuales en su mayoría se encuentran en las partes sur y oeste de esta isla, aunque el área habitacional se extiende por las áreas circunvecinas a los lagos.

Se ha encontrado en la superficie fragmentos de estelas esculpidas en roca caliza dura y que, según Ekholm (1979: 147), no pertenecen al estilo maya clásico de las tierras bajas; también existen bajorrelieves que representan caras o soles en rocas, aunque los fragmentos de piedra trabajada que hemos hallado muestran elementos decorativos bien definidos (ver posteriormente su descripción cuando se trata el montículo tres).

Al oeste de la isla El Limonar está la del Cangrejo (figura 2), donde sólo queda un montículo en su extremo norte. En la isla El Sabino hay dos pequeños montículos y en su extremo sur se localiza toda una sección con grandes montículos que conforman una pequeña plaza. La siguiente isla hacia el norte no tiene nombre local y actualmente es utilizada para el cultivo y sólo se puede apreciar en la superficie gran cantidad de material cerámico. Siguiendo al noroeste hay otra pequeña isla sin nombre con tres pequeños montículos, y, junto con la que le sigue a la izquierda en la zona de Las Cascadas, aún tiene vegetación primaria, por lo que las estructuras todavía están conservadas. Hay otra sección al noroeste, conocida por los lugareños como Los Gemelos, llamada así por encontrarse dos montículos del mismo tamaño 
y por estar cerca del camino vecinal que va del ejido Cristóbal Colón a Rodulfo Figueroa, pero han sido parcialmente destruidos. Al noroeste del sitio se localiza otra sección llamada El Chicozapote, en la que los montículos todavía están protegidos por la vegetación tropical, igual que los de la isla El Hasam (Figura 2). En la parte central del sitio Lagartero (norte de la isla El Limonar) se encuentran las islas de Puente Mora y El Búho con las secciones aledañas, donde afortunadamente hay vegetación, por lo que las estructuras están bien preservadas.

\section{Trabajo arqueológico}

En la primera temporada de campo, 1990, se hizo el recorrido completo del sitio y el levantamiento detallado de El Limonar (figura 3). Se excavó la entrada suroeste a la isla, en la que fueron encontrados los cimientos de dos estructuras de una y dos hiladas de piedra trabajada, pero no se halló división interna alguna, ni piso; tenían una planta rectangular y se les volvió a cubrir con la misma tierra de la excavación por haberse encontrado muy destruidas. Aquí fueron halladas seis vasijas rotas. Lo más importante de esta temporada fue que con una parte del presupuesto asignado, el Instituto Nacional de Antropología e Historia (INAH) adquirió toda la isla El Limonar.

En la segunda temporada de campo, 1993, se comenzó a explorar el juego de pelota localizado en la parte media de la isla El Limonar (figura 3); solamente fue liberada la estructura sur, de planta rectangular ( $27.5 \times 13$ metros) con una escalera de acceso de cinco escalones por el lado sur y una altura de 2.5 metros (figura 4).

En la tercera temporada de campo, 1994, se excavó la estructura norte del Juego de Pelota (figura 4), que tiene el mismo tamaño y forma que los del sur y también con una escalera de acceso, por su lado norte, con cinco escalones. Se comenzó con la exploración de los cabezales este y oeste (figura 4), y fueron halladas mediante este proceso cinco altares directamente al sur de la estructura sur.

En la cuarta temporada de campo, 1995, se consolidó tanto las estructuras sur y norte del Juego de Pelota como la parte liberada de los cabezales este y oeste.

En la quinta temporada, 1996, los extremos este y oeste de los cabezales del Juego de Pelota fueron liberados para definir si éste fue cerrado o abierto; y resultó ser del primer tipo (foto 3). Cada cabezal tiene respectivamente en sus extremos este y oeste una estructura rectangular, con escalones para entrar y salir de la cancha (Rivero, 1996); la mejor conservada es la del lado este; presentando por el lado oeste una escalera con dos escalones limitados por alfardas y por el este con dos grandes peldaños en cada extremo de la estructura. También se exploró el montículo uno, que limita a la plaza principal por el lado sur y está al norte del Juego de Pelota (figura 3). Hasta donde se pudo liberar, es un basamento piramidal de seis cuerpos; al parecer todos los muros fueron rectos, con excepción del primero al tercero, con una escalera de acceso por el lado norte. Tuvo dos épocas constructivas, hizo falta explorar el lado norte y consolidarlo (figura 5).

En la sexta temporada, 1997, se terminó de excavar el lado norte de la pirámide uno y se le consolidó, y el resultado fue una estructura de siete cuerpos. En la primera etapa constructiva los primeros tres y el penúltimo tenían talud y tablero, el cuarto y quinto eran rectos; la primera escalera terminaba al comienzo del sexto cuerpo; en la segunda etapa se agregó tres escalones en el lado sur sobre el talud del primer cuerpo. La 
segunda escalera, colocada sobre la primera, llega hasta el pequeño muro recto que está por encima del sexto cuerpo; a la escalera se le añadieron las banquetas y las dos rampas laterales (Rivero, 1999), que están por encima del segundo y tercer cuerpos. El quinto cuerpo del lado sur presenta como decoración un diseño doble en forma de $\mathrm{T}$ invertida, símbolo del Dios del Viento (figura 6); por el lado norte, este mismo cuerpo tiene cuatro páneles paralelos, dos salidos y dos remetidos, intercalados, dando así un contraste de claroscuro (Rivero, 1999: 56-61; figura 7). Esta pirámide tiene una altura desde la base de 6.50 metros y una base de 27 por los lados sur y norte y 24 por los del lado este y el oeste y una rampa por afuera del primer cuerpo de siete metros en la última etapa constructiva (foto 4). También se exploró y consolidó un pequeño montículo, el primero, junto a la pirámide uno en el lado sureste (Figura 3), el cual se encontró intacto, resultó ser un altar con una estructura de tres cuerpos rectos, de planta rectangular y con una pequeña escalera de tres escalones por el lado este, limitada por alfadas (figura 7); tiene una altura de $1.60 \mathrm{~m}$. y una base de $6.60 \times 4$ (foto 5).

En la séptima temporada de campo, 1999, se liberó y consolidó el cuarto montículo, localizado muy cerca del 4 (figura 3), al sur de éste. Se trata de una estructura de planta rectangular, $14.60 \mathrm{x}$ 7.50 metros, con una escalera de acceso por el lado sur de cinco escalones limitados por alfardas y una altura de 1.50 metros (Foto 7). En la parte media de la escalera presenta una decoración en forma de cuadro (figura 8).

Fueron terminados de explorar y consolidar los cinco altares localizados inmediatamente al sur del Juego de Pelota, de los cuales solamente se conservan los cimientos; tienen todos formas y tamaños diferentes. Describiendo de este a oeste, tenemos el altar uno, de forma rectangular, con paredes en talud, dos escalones de acceso por el lado este, que mide 6 × 4 metros y 30 centímetros de altura. El altar dos también presenta una planta rectangular con muros en talud. No fueron encontrados escalones de acceso, los cuales por la inclinación y el deterioro de su superficie pudieron haber estado en el lado sur. Mide 6.20 × 4.50 metros, con una altura de 40 centímetros. El altar tres tiene base rectangular, muros en talud, tres escalones tanto por el lado este como por el oeste y mide 8.15 x 5.20 metros con una altura de 70 centímetros. El altar cuatro tiene cimientos rectangulares. Se le encontró bastante destruido; conserva sólo el muro del lado este, y es el más pequeño de todos, pues mide 3.20 × 2.10 metros con una altura de 30 centímetros. El altar cinco es el más completo y grande de todos; mide $10.20 \times 7.35$ metros con una altura de 1.50. Está formado por dos cuerpos y probablemente tuvo tres escalones de acceso por el lado sur y el muro del segundo cuerpo es en talud (figura 9).

La octava temporada de campo fue dividida en varias etapas. En la primera, 2000, se exploró el montículo cinco, localizado al oeste de la estructura cuatro y del Juego de Pelota (figura 3), el que al liberarlo mostró que no era un montículo propiamente hablando sino el conjunto de ocho basamentos habitacionales, los cuales estaban en mal estado de conservación por encontrarse en el área de paso de los ejidatarios que entraron a la isla; además, como era una prominencia baja, también fue arado para cultivar la tierra. No obstante, los basamentos fueron consolidados hasta donde se hallaron evidencias.

El más grande de los cimientos tiene base rectangular y mide $8.50 \times 6$ metros con una altura de 60 centímetros; presenta muros en talud con 
cinco escalones de acceso por el lado este; al sur hay junto a él otro basamento rectangular bastante destruido de menor tamaño y también con muros en talud; quedan sólo visibles los lados oeste y sur. Junto a éste se encuentra otro cimiento cuadrangular más pequeño de paredes rectas unido por una hilera de piedras. Al oeste de este basamento se encuentra otro cimiento de casa de forma rectangular con muros en talud y con tres escalones que dan al lado sur. Al norte de éste se halla otro también en forma rectangular con muros en talud, remetidos en las esquinas norte y sur; presenta tres escalones del lado oeste, los cuales dan a un pequeño patio. Al norte hay otros basamentos rectangulares sin restos de escalones. Por último, en el extremo suroeste se conservaron solamente los muros norte y este de otro cimiento y al oeste los muros sur y este de otro basamento de casa, quedaron sólo los restos de dos hiladas de piedra de una escalera mal conservada por el lado este (figura 10 y foto 7).

En la segunda etapa, 2001, se excavó y restauró el montículo tres, que limita fa plaza principal por el lado norte (Figura 3). Es una pirámide de siete cuerpos rectos y una escalera por el lado sur (foto 6) limitada por alfardas; es un poco mayor que la pirámide uno, con una altura de siete metros y una base de veinte metros cuadrados (figuras $11 \mathrm{y}$ 12). Las otras dos pirámides que limitan la plaza por los lados oeste y este (2 y 4) todavía no han sido exploradas; la dos es la más alta de todas. En la liberación de la pirámide tres, además de haberse encontrado cerámica y lítica, fueron hallados dos fragmentos de estelas. Uno representa un cráneo en posición lateral con un glifo y cuatro numerales (figura 13) y el otro muestra una serie de círculos unidos (figura 14). Al limpiar al este del cabezal este del Juego de Pelota, se localizó un muro bien conservado, con una altura de 1.20 metros, el cual por estar al nivel del cabezal permanecía oculto.

En la tercera y última fases de la octava temporada de campo, 2003, se terminó de liberar y restaurar la estructura al oriente del cabezal este del Juego de Pelota; y una tiene base rectangular con una altura por el lado oeste de 1.20 metros y por el este de 50 centímetros, con una base de 11.10 x 5 metros y una escalera por el lado este de tres escalones (figuras 15 y 16 y foto 8). También se excavó y consolidó la estructura dos, localizada en el lado noreste del montículo dos (figura 3), la que por su tamaño y cercanía con éste pudo haber funcionado como altar en un momento dado. Es una estructura que tuvo dos etapas constructivas, en la primera los muros eran en talud y tablero y la escalera estaba formada por tres grandes peldaños que daban al lado oeste con una base de 11 x 8 metros y una altura de 1.30 (figuras 17 y 18 $y$ foto 9). En la segunda etapa ésta fue de nuevo reocupada, se construyó sobre ella otra estructura de tres cuerpos. El primero es de muros rectos terminados en cornisas y una escalera también por el lado oeste con tres escalones limitados por anchas alfardas decoradas en su parte inclinada por grandes lajas estucadas. El segundo cuerpo, hasta donde se pudo apreciar, tiene paredes rectas y por el mismo lado presenta otra escalera de tres peldaños con una decoración, que por primera vez se encuentra en el sitio, de cuatro grandes lajas verticales inclinadas en la parte media de ella, como también en los extremos de cada lado de la escalera hay lajas más pequeñas que las de la parte media. El tercer y último cuerpo tiene los muros rectos y está menos conservado, por lo que es de menor tamaño que el primero y el segundo (foto 10). En total tiene una base de 15.30 x 17.20 metros, incluida la longitud de las alfardas, y una altura de 2.50 por el lado este y 2.30 por el oeste (figuras 19 y 20). 
Acerca de la cronología de las estructuras trabajadas, con base en la cerámica y el estilo arquitectónico, podemos decir que la primera etapa constructiva de la pirámide uno, el Juego de Pelota con sus cinco altares asociados, la primera etapa constructiva de la estructura cuatro y los ocho basamentos de casa y la primera etapa constructiva de la estructura dos pertenecieron al Clásico Tardío (750 - 900 d. C.). La segunda y última etapa constructiva de la pirámide uno, de las estructuras cuatro y dos, como la que se encuentra al oriente del cabezal este del Juego de Pelota, el altar uno y la pirámide tres arrojan fechas dentro del lapso que va del 900 al $1200 \mathrm{~d}$. C., lo que correspondería al Posclásico Temprano.

\section{Comentarios adicionales}

El sitio arqueológico de Lagartero, según la información obtenida mediante las excavaciones, fue habitado principalmente del Clásico Tardío, 750 a 900 d. C., al Posclásico Temprano, 900 a 1200 d. C., por gente que habló una lengua de la rama oeste del grupo mayense (Kaufman, 1974: 85) -de la que aún queda el jacalteco, actualmente, la lengua etnográfica más cercana al sitio-, asentada en pequeñas islas donde construyeron estructuras habitacionales y ceremoniales, aprovechando su localización privilegiada en un pequeño microambiente en la región de tierra caliente; utilizaron para su subsistencia tanto el medio terrestre como el lacustre, por lo que fue una zona rica en alimentos. Esta abundancia de productos alimenticios permitió que desarrollaran el trabajo arquitectónico, como en cantidad y calidad de los restos materiales que produjeron, ya que aunque solamente se ha excavado una mínima parte del área ceremonial en estas estructuras se ha recuperado una serie de vasos policromos estilo códice del Clásico Tardío, 600-900 d. C., (Rivero, 2003), decorados con personajes ricamente ataviados, generalmente en posición sedente con la vírgula de la palabra y una serie de seudoglifos - se les llama así por presentar en lo general elementos repetitivos-, como ollas policromas, vasijas bicromas y monocromas de diferentes tamaños y una variedad de figurillas antropomorfas y zoomorfas modeladas y moldeadas (Rivero, 2002), además de artefactos trabajados en diferentes tipos de rocas (Rivero, 1996: 498-532). Todo esto demuestra el grado de especialización de los artesanos que habitaron en este lugar en esos remotos tiempos, como también sus nexos comerciales con los Altos de Guatemala, principalmente para el Posclásico Temprano con los tipos cerámicos Chinautla Policromo y Plombe, así como para el Clásico Tardío con la obsidiana verde que procede del Cerro de las Navajas en el centro de México.

\begin{tabular}{|c|c|c|c|c|}
\hline \multicolumn{5}{|c|}{ Cuadro cronológico de las estructuras trabajadas en el área del Limona } \\
\hline Estructura & $\begin{array}{l}\text { No. De etapas } \\
\text { constructivas }\end{array}$ & \multicolumn{3}{|c|}{ Períodos } \\
\hline Juego de Pelota & 2 & $\begin{array}{l}\text { Clásico Medio } \\
\text { (500-700 d. C.) }\end{array}$ & Clásico Tardío & \\
\hline $\begin{array}{l}\text { Pirámide } \\
\text { número } 1\end{array}$ & 2 & $\begin{array}{l}\text { Clásico Medio } \\
(500-700 \text { d. C. })\end{array}$ & $\begin{array}{l}\text { Clásico Tardío } \\
\text { (700-900 d. C) }\end{array}$ & \\
\hline Altar 1 & 1 & & $\begin{array}{l}\text { Clásico Tardío } \\
\text { (700-900 d. C) }\end{array}$ & \\
\hline Altar 4 & 1 & & $\begin{array}{l}\text { Clásico Tardío } \\
\text { (700-900 d. C) }\end{array}$ & \\
\hline Pirámide no. 3 & 2 & & $\begin{array}{l}\text { Clásico Tardío } \\
\text { (700-900 d. C) }\end{array}$ & $\begin{array}{c}\text { Posclásico } \\
\text { Temprano } \\
\text { (900-1200 d. C) }\end{array}$ \\
\hline Montículo no. 5 & 1 & & $\begin{array}{l}\text { Clásico Tardío } \\
\text { (700-900 d. C) }\end{array}$ & \\
\hline $\begin{array}{l}\text { Cinco altares al } \\
\text { sur del Juego de } \\
\text { pelota }\end{array}$ & 1 & & $\begin{array}{l}\text { Clásico Tardío } \\
\text { (700-900 d. C) }\end{array}$ & \\
\hline $\begin{array}{l}\text { Estructura al } \\
\text { este del Juego de } \\
\text { pelota }\end{array}$ & 1 & & $\begin{array}{l}\text { Clásico Tardío } \\
\text { (700-900 d. C) }\end{array}$ & \\
\hline
\end{tabular}




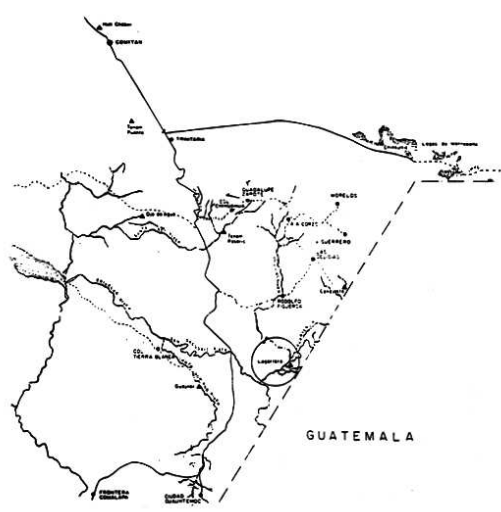

Figura 1: localización del sitio arqueológico de Lagartero, mpio. de La Trinitaria, Chiapas.

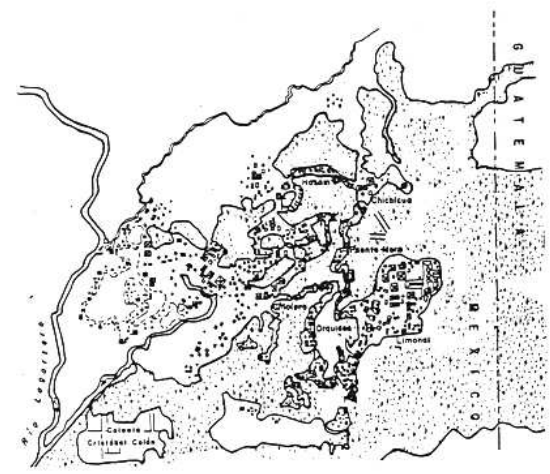

Figura 2: croquis de Lagratero y plano del sitio.

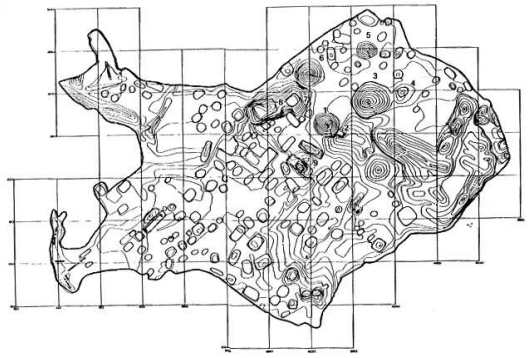

Figura 3: ubicación de los montículos localizados dentro de la isla El Limonar.

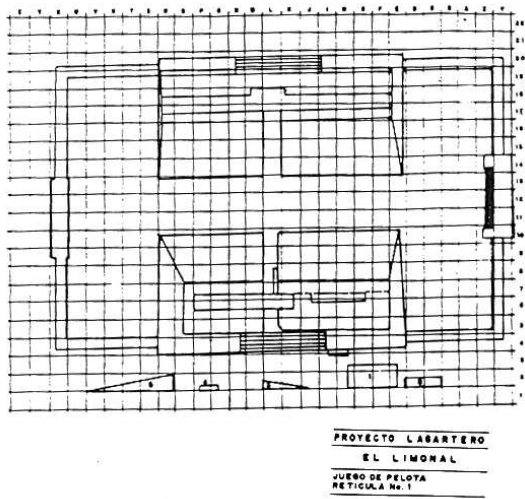

Figura 4: juego de pelota ubicado en la isla El Limonar.

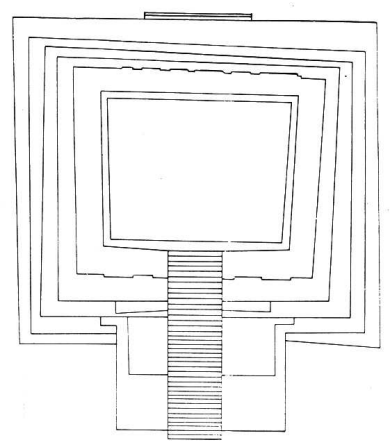

Figura 5: planta del montículo 1 ya excavado.

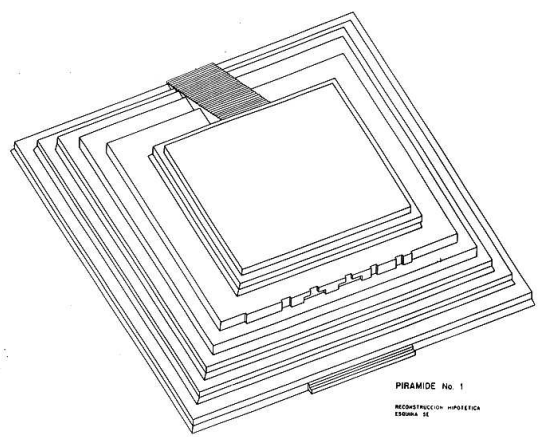

Figura 6: pirámide 1, reconstrucción hipotética. 


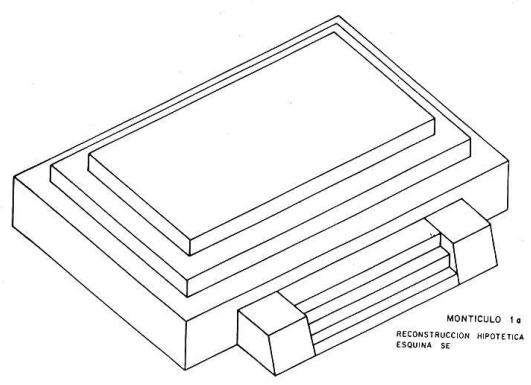

Figura 7: reconstrucción del montículo 1a.

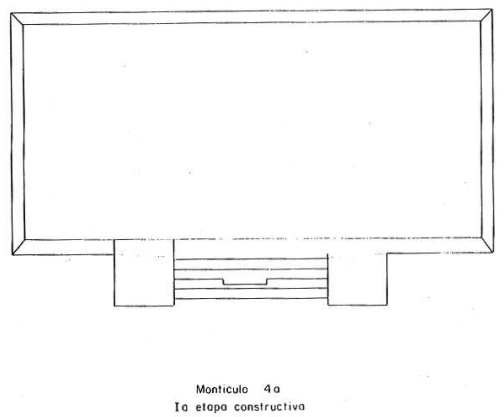

Figura 8: primera etapa constructiva del montículo 4.

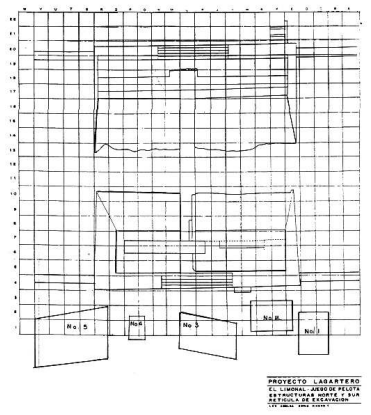

Figura 9: altares localizados al sur del juego de pelota.

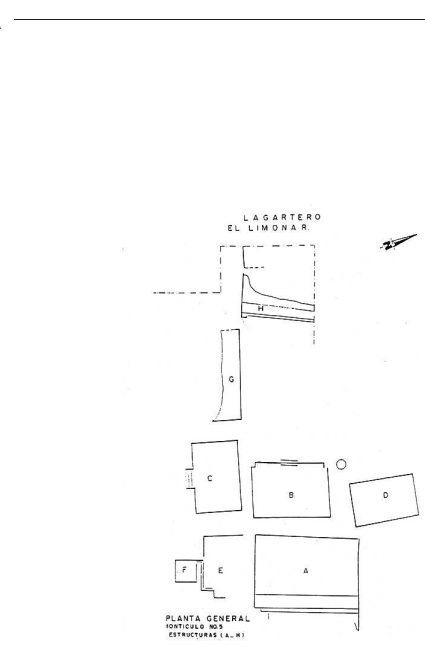

Figura 10: planta general del montículo 5.

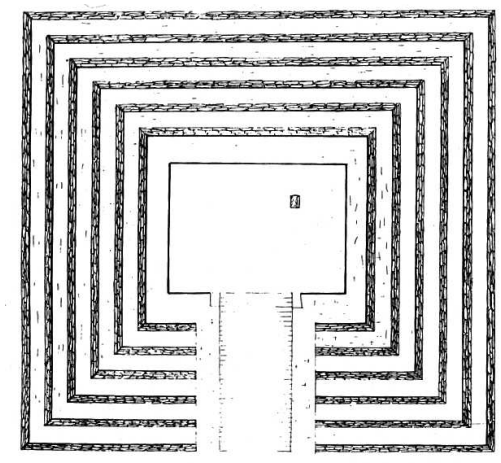

Figura 11: planta general del montículo 3.

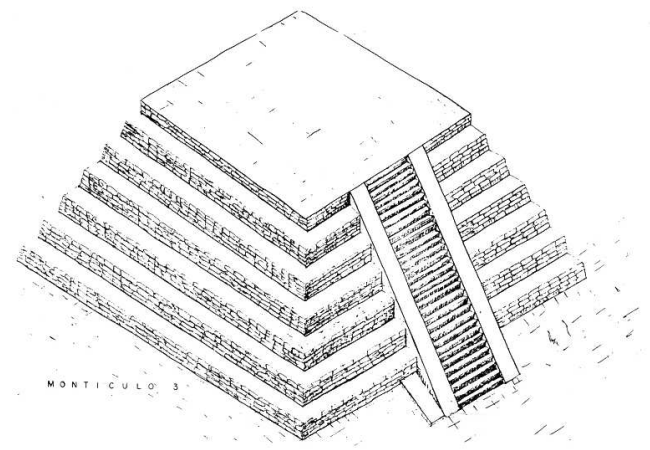

Figura 12: montículo 3 ya explorado.

\section{1}

Revista LiminaR. Estudios sociales y bumanísticos, año 5, vol. V, núm. 1, junio de 2007, Tuxtla Gutiérrez, Chiapas. ISSN: 1665-8027 


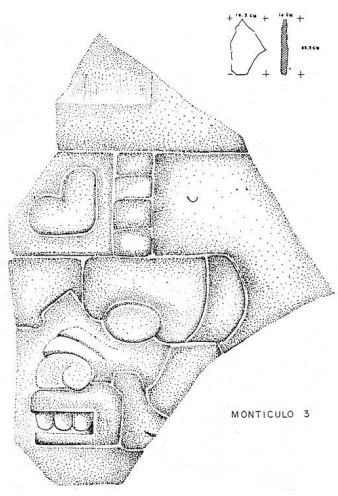

Figura 13: fragmento de estela localizado en el montículo 3.
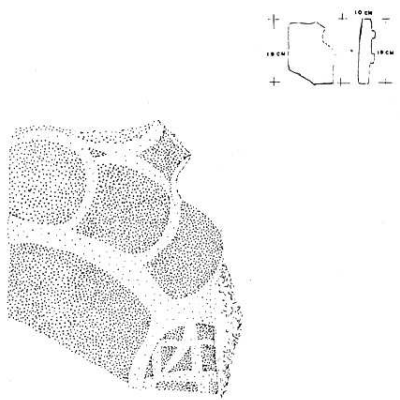

MONTICULO 3

Figura 14. fragmento de estela localizado en el montículo 3, muestra círculos.

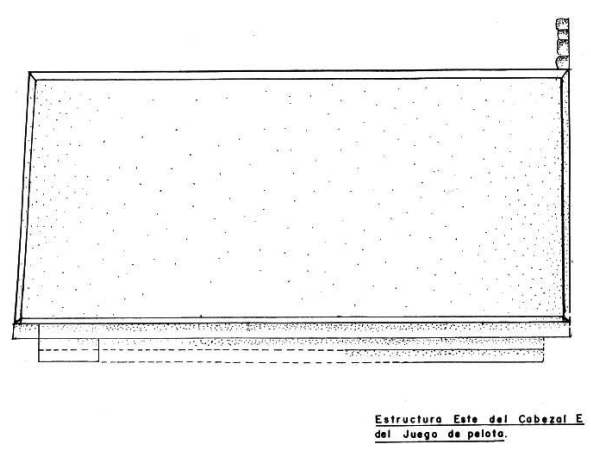

Figura 15: estructura este del cabezal E del juego de pelota.

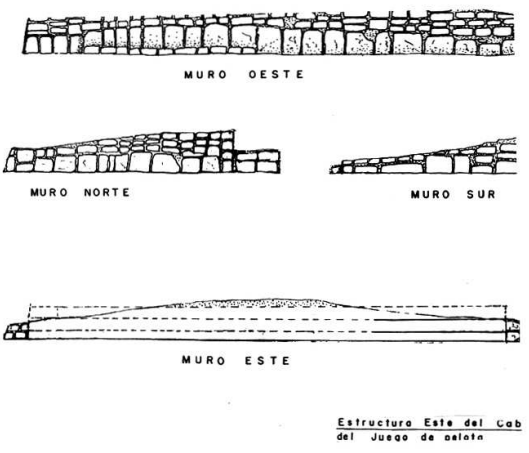

Figura 16: muros liberados de la estructura este del cabezal E.

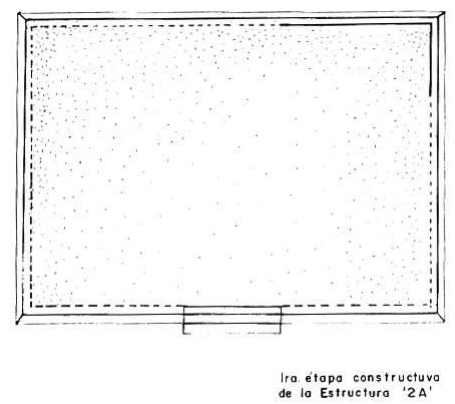

Figura 17: primera etapa constructiva de la estructura $2 \mathrm{~A}$.

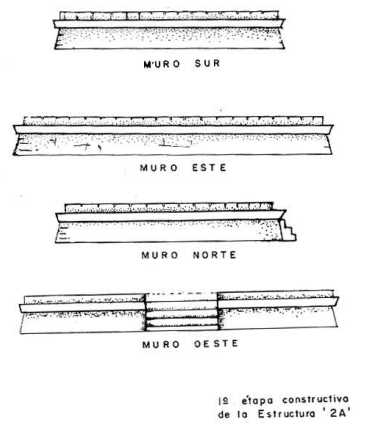

Figura 18: perfiles de los muros. 


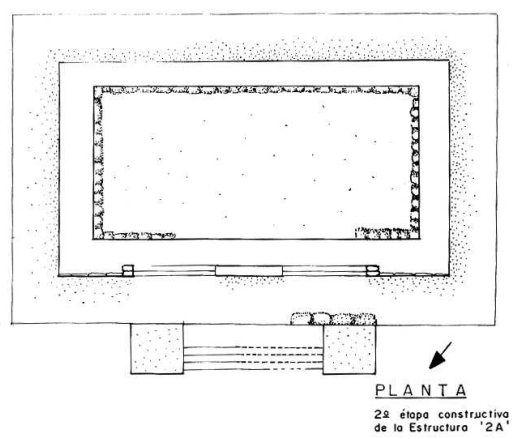

Figura 19: planta de la segunda etapa constructiva de la estructura 2A.

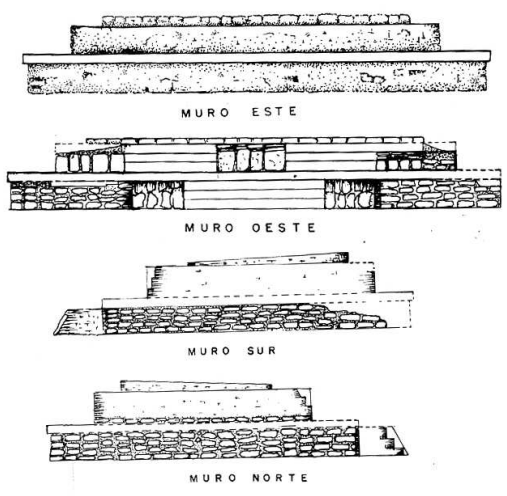

Figura 20: perfiles de los cuatro muros.

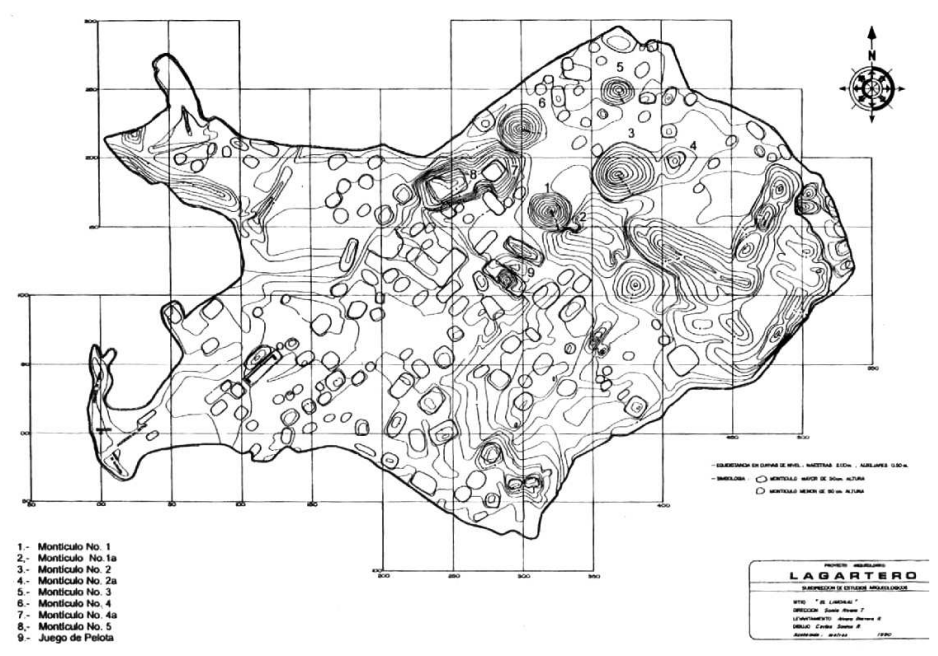

Isla 


\section{Bibliografía}

Ekholm, Susana M., 1979, "Lagartero figurine", en Maya Archaeology and Ethnology, editado por Norman Hammond y Gordon R. Willy, Universidad de Texas Press, Austin y Londrespp. 172-189.

,1979b, The significance of an extraordinary maya ceremonial refuse deposit at Lagaratero, Chiapas”, Actes du XLII Congrés International des Americanists, Vol. VIII, pp. 147-159. Societé des Americanistes, Musée d'Homme, Paris.

, 1980, "The Lagartero ceramic pendants", Fourth Palenque Round Table, , Vol. 6, pp.211-219, Precolumbian Art Research Institut.

Gurr, Deann, 1987, The burial patterns of Lagartero, Chiapas, tesis doctoral, Universidad de Utah, Salt Lake City, USA..

Kaufman, T., 1974, Idiomas de Mesoamérica, editado por José de Pineda Ibarre, ministro de Educación, Guatemala.

Lee, Thomas A. Jr., 1974, Preliminary report of the $2^{\text {nd }}$ and final reconnaissance season of Uper Grijalva Basin Maya Proyect, enero a mayo de 1974, manuscrito inédito entregado al Consejo de Arqueología del INAH.

Martínez, Eduardo, 1983, "Lagartero: una situación única de la cuenca superior del río y S. M. Ekholm, Grijalva”, en Antropología e historia de los mixezoque, en homenaje a Frans Blom, editado por Lorenzo,.Ochoa y Thomas Lee Jr., pp. 225-270, Centro de Estudios Mayas, UNAM y NWAF.
Miranda, Faustino, 1976, La vegetación de Chiapas, edición del Gobierno del Estado de Chiapas, Tuxtla Gutiérrez, Chiapas.

Müllerried, Federico, 1957, Geología de Chiapas, Gobierno Constitucional del Estado de Chiapas, Tuxtla Gutiérrez, Chiapas, México.

Rivero Torres, Sonia, 1996, "El Juego de Pelota del sitio Lagartero, Chiapas", Quinto Foro de Arqueología de Chiapas, Gobierno del Estado de Chiapas, UNACH, pp. 39-52. , 1996, La lítica del Juego de Pelota de Lagartero, Chiapas", en el Anuario del Centro de Estudios Superiores de México y Centroamérica. UNICACH., pag: 498-533. , 1997, "La cerámica y lítica de Lagartero, Chiapas, procedente de El Limonar, Unidad 1", en Homenaje al profesor César A. Sáenz, Colección Científica No. 351, pp. 201-250, INAH. , 1999, "Montículo 1 del Sitio Arqueológico de Lagartero, municipio La Trinitaria, Chiapas, México", en México, Vol. XXI, No. 3, pp. 58-61. ,2002, Figurillas antropomorfas y zoomorfas del juego de pelota de Lagartero, Chiapas, Serie Ciencias Sociales, y Humanidades de la UNICACH, Tuxtla Gutiérrez, Chiapas.

,2003, "Cuatro vasos policromos de Lagartero, Chiapas”, revista Arqueología del INAH, enerojunio, no. 29.

Willey, G. P. et al., 1967, "Maya lowland ceramic. A report from the 1965 Guatemala City Conference", American Antiquity, Vol. 32, No.3, pp. 289-305. 